\title{
Health-System Introductory Pharmacy Practice Experience as an online course in the United States
}

\author{
M. Kawaguchi-Suzuki*, J. Merlo, S. White, R. Gibbard, L. Ly, A. Bzowyckyj, D. Bojorquez, J. Begert, E. Saito, K. Marcus \\ Pacific University School of Pharmacy, USA.
}

\section{Keywords}

Experiential Education

IPPE

Pharmacy

Online Learning

USA

\section{${ }^{*}$ Corresponding author: marina.suzuki@pacificu.edu}

\begin{abstract}
Summary: Introductory Pharmacy Practice Experiences (IPPEs) provide early experiential education in the Doctor of Pharmacy (Pharm.D.) curriculum in the United States (US). In response to Oregon's 'Stay Home, Save Lives' executive order issued during the COVID-19 pandemic, an online health-system IPPE course was developed to simulate the practice experiences that have historically been conducted in person. This case study describes experience from the online health-system IPPE course offered for incoming second-year student pharmacists enrolled in a three-year Pharm.D. programme at Pacific University in Oregon, US. The goals of the course were: 1) to expose students to pharmacy practice common in health-system settings in the US; and 2) for students to earn 50 experiential clock hours through simulation activities.
\end{abstract}

\section{Background and Context}

Introductory Pharmacy Practice Experiences (IPPEs) take place early in the Doctor of Pharmacy (Pharm.D.) curriculum in the United States (US) with a minimum of 300 clock hours required per the 2016 standards set by the Accreditation Council for Pharmacy Education (ACPE) (ACPE, 2015). The goal of the IPPE is to expose students to common practice models, typically with students training alongside pharmacists. The required hours are balanced between community and health-system settings.

On 8th March 2020, the Governor declared a state of emergency in Oregon to address the spread of COVID-19, and the 'Stay Home, Save Lives' executive order was issued (Office of Governor State of Oregon, 2020). During the early pandemic, it was challenging for students to visit healthsystem sites for their regularly scheduled IPPEs due to issues with site capacities, the need to conserve personal protective equipment, social distancing requirements, and concerns about student on-boarding in the midst of increasing local COVID-19 cases (Oregon Health Authority, 2020). In response to the inability to have students placed at health-system sites, an online course was developed to provide learning activities similar to IPPEs since the ACPE allows simulated practice experiences to count for a maximum of 60 clock hours (ACPE, 2015). Since the on-site component was delayed to future dates, it was essential to bridge didactic classroom learning to actual practice for students' professional development. This case study shares experiences from an online health-system IPPE course offered for incoming second-year students enrolled in a 3-year Pharm.D. programme at Pacific University in Oregon.

\section{Educational Description}

The health-system IPPE was conducted during the summer following completion of the first year of the 3-year Pharm.D. curriculum. A seven-week, fully online course was offered to 95 students. The course goals were: 1 ) to expose students to health-system pharmacy practice models through virtual shadowing days; and 2) to provide 50 experiential clock hours through asynchronous simulated practice activities. Two faculty coordinators developed the course with assistance from eight preceptors with clinic or hospital practice experience. Technologies utilised in the course included an online learning management system (Moodle), a video 
communication platform (Zoom), and a video sharing platform (Flipgrid). All students were provided with individual access to an electronic health record (Epic) populated with simulated patient charts.

The simulation activities were asynchronous individual assignments emulating pharmacist-delivered patient care situations. These included chart reviews, medication reconciliation, verifying prescriptions, utilising hospital protocols, primary literature analyses, calculations, formulary therapeutic interchanges, discharge counselling, and drug information (DI) question responses. For example, the DI assignment provided background patient information, the encounter setting, and a question from a healthcare professional. Students video-recorded themselves responding to the question. Simulation activities were supplemented with learning modules covering medical terminology, common medication errors, and interpretations of lab values. Faculty-hosted synchronous sessions were used for student questions and activity guidance.

Shadowing days were designed for students to meet and discuss pharmacy practice models with different preceptors through synchronous Zoom sessions. Preceptors shared their professional backgrounds and practice settings, including both acute and ambulatory care. For consistency between sessions, preceptors utilised a slide template with prompts to guide a discussion of their practice environments, patient care workflows, and interactions with other healthcare professionals. In addition to showcasing the preceptor's practice model, each session had a different theme to connect students' simulation activities to real-world examples of pharmacy practice. These themes included an overview of health-systems, working-up patients for inpatient rounds or clinic appointments, the pharmacists' patient care process, staffing the central pharmacy, medication reconciliation, curb side consultations, affordable care for patients, population management, DI responses, and drug policy.

Additionally, students submitted a weekly video reflection commenting on what they learned, skills they developed, challenges they faced, and skills that needed more practice. The Pharm.D. programme employs a competency-based Pass/No Pass grading system. To pass this course, students were required to participate in the shadowing sessions, successfully complete all simulation activities, and pass an open-book online final exam. After the course, students were asked to submit a course evaluation.

\section{Outcomes and Recommendations}

This online IPPE course allowed students to learn about health-system pharmacy practice and earn 50 experiential hours. Sixty out of 95 students submitted feedback; the overall course evaluation was favourable (weighted mean of 4.16 on a scale from 0 to 5 ).
One limitation was that students expressed disappointment at not being physically at a health-system site. The intent of the course was not to replace the entire IPPE, but to bridge their classroom learning to future on-site experiences. Despite not earning any experiential hours from the virtual shadowing days, students highly valued the live interactions to learn how their knowledge and skills apply in practice through preceptors' demonstrations and activities. Another limitation was faculty workload. While the weekly reflection allowed frequent check-ins and ongoing evaluation of the course, continuous adaptation was required. With a limited number of faculty, feedback was provided globally rather than on an individual basis. This empowered students to assess their own learning while reducing the grading burden on faculty.

One recommendation is to set a regular schedule for synchronous sessions and asynchronous assignments so that students can create their own routine. It is essential to clearly communicate course organisation and assignment deadlines. The second recommendation is to make the recordings of synchronous sessions available for students who cannot participate at scheduled times. During the pandemic, some students had to adjust to dynamic living situations, and attending synchronous sessions was not always possible. In these cases, the recorded videos were made available, and students completed make-up assignments.

Based on each students' performance and feedback, the coordinators and preceptors agreed that the online IPPE course provided a unique learning opportunity that exposed students to health-system pharmacy practice and prepared them for future coursework and on-site experiential education.

\section{References}

ACPE [Accreditation Council for Pharmacy Education]. (2015). Accreditation Standards and Key Elements for the Professional Program in Pharmacy Leading to the Doctor of Pharmacy Degree. Available at: https://www.acpe-accredit.org/pdf/Standards2016 FINAL.pdf

Office of Governor State of Oregon. (2020). Executive Order No. 20-12. Available at: https://www.oregon.gov/gov/Documents/ executive orders/eo 20-12.pdf

Oregon Health Authority. (2020). COVID-19 Updates. Available at: https://govstatus.egov.com/OR-OHA-COVID-19 